\title{
Structure and Evolution of Supergranulation from Local Helioseismology
}

\author{
Johann Hirzberger • Laurent Gizon • Sami K. Solanki • \\ Thomas L. Duvall, Jr.
}

Received: 21 October 2007 / Accepted: 12 May 2008 / Published online: 1 July 2008

(C) The Author(s) 2008. This article is published with open access at Springerlink.com

\begin{abstract}
Supergranulation is visible at the solar surface as a cellular pattern of horizontal outflows. Although it does not show a distinct intensity pattern, it manifests itself indirectly in, for example, the chromospheric network. Previous studies have reported significant differences in the inferred basic parameters of the supergranulation phenomenon. Here we study the structure and temporal evolution of a large sample of supergranules, measured by using local helioseismology and SOHO/MDI data from the year 2000 at solar activity minimum. Local helioseismology with $f$ modes provides maps of the horizontal divergence of the flow velocity at a depth of about $1 \mathrm{Mm}$. From these divergence maps supergranular cells were identified by using Fourier segmentation procedures in two dimensions and in three dimensions (two spatial dimensions plus time). The maps that we analyzed contain more than $10^{5}$ supergranular cells and more than $10^{3}$ lifetime histories, which makes possible a detailed analysis with high statistical significance. We find that the supergranular cells have a mean diameter of $27.1 \mathrm{Mm}$. The mean lifetime is estimated to be 1.6 days from the measured distribution of lifetimes (three-dimensional segmentation), with a clear tendency for larger cells to live longer than smaller ones. The pair and mark correlation functions do not show pronounced features on scales larger than the typical cell size, which suggests purely random cell positions. The temporal histories of supergranular cells indicate a smooth evolution from their emergence and growth in the first half of their lives to their decay in the second half of their lives (unlike exploding granules, which reach their maximum size just before they fragment).
\end{abstract}

Keywords Sun: supergranulation · Sun: photosphere $\cdot$ Helioseismology

Helioseismology, Asteroseismology, and MHD Connections

Guest Editors: Laurent Gizon and Paul Cally

J. Hirzberger $(\bowtie) \cdot$ L. Gizon · S.K. Solanki

Max-Planck-Institut für Sonnensystemforschung, 37191 Katlenburg-Lindau, Germany

e-mail: hirzberger@mps.mpg.de

T.L. Duvall, Jr.

Laboratory for Solar and Space Physics, NASA Goddard Space Flight Center, Greenbelt, MD 20771, USA 


\section{Introduction}

The velocity field in the solar photosphere is dominated by turbulent convective motions. These motions manifest themselves as two main horizontal scales - granulation and supergranulation. The existence of a distinct intermediate scale of mesogranulation is still under debate (see, e.g., Rieutord et al., 2000). The granular convective scales $(\approx 1.5 \mathrm{Mm})$ are directly observable in brightness as a cellular pattern, which is closely related to the vertical motion of the gas determined from the spectral line shifts. Supergranular structures $(\approx 30 \mathrm{Mm})$ are easily detectable away from the center of the solar disk in maps of the lineof-sight Doppler velocity (e.g., Hathaway et al., 2000): Supergranulation is associated with excess power near spherical harmonic degree $\ell=120$. Supergranulation is outlined by the network-like distribution of quiet-Sun magnetic fields, visible as chromospheric emission in the Ca II H and K spectral lines. Supergranules, however, are barely detectable in intensity. Elaborate measurements by Meunier, Tkaczuk, and Roudier (2007) show a small intensity contrast that would correspond to a temperature difference of $0.8-2.8 \mathrm{~K}$ between cell centers and boundaries; these results are compatible with a convective origin of supergranulation. Supergranulation can also be detected indirectly by tracking the proper motions of surface features such as granules or magnetic bright points (e.g., Leighton, 1964; Rimmele and Schröter, 1989; Shine, Simon, and Hurlburt, 2000; DeRosa and Toomre, 2004; Rieutord et al., 2007).

Since the discovery of supergranulation (Hart, 1956; Leighton, Noyes, and Simon, 1962) several attempts have been made to explain its origin. Simon and Leighton (1964) proposed that a local minimum of the vertical adiabatic temperature gradient in the deep solar layers caused by $\mathrm{He}$ I or He II ionization would favor convective cells with horizontal sizes comparable to the depth of the ionization zones. Alternatively, supergranulation may be related to the spatial ordering of smaller convective cells. For example, Ploner, Solanki, and Gadun (2000) showed that mesogranular-scale structures are formed in two-dimensional (2D) simulations even if these are not sufficiently deep to reach the ionization zones. Rieutord et al. (2000) and Roudier et al. (2003) suggested that a pattern at supergranular scales could emerge from the nonlinear collective interaction of granules. Using a numerical $n$-body diffusion-limited aggregation model, Crouch, Charbonneau, and Thibault (2007) showed that the advection of magnetic elements by the granular flow may produce a supergranular network. The same simulation suggests that the supergranular cell size decreases with magnetic activity, which has recently been observed by Meunier, Roudier, and Tkaczuk (2007).

Various techniques of local helioseismology have been used to infer supergranular flows just below the photosphere (e.g., Kosovichev and Duvall, 1997; Duvall and Gizon, 2000; Woodard, 2002; Zhao and Kosovichev, 2003; Braun, Birch, and Lindsey, 2004; Gizon and Birch, 2005, and references therein). Using $f$-mode time - distance helioseismology, Duvall and Gizon (2000) mapped supergranular flows and studied their evolution. This method of analysis provides information about the two horizontal components of velocity and the horizontal divergence of the flow. Duvall and Gizon (2000) described the distribution of the divergence values and noted that the spatial power spectrum of the divergence maps peaks at spherical harmonic degree 120. Using the same $f$-mode data, del Moro et al. (2004) found supergranular diameters in the range 10-40 Mm and determined a mean diameter of $27 \mathrm{Mm}$. Beck and Duvall (2001) measured the autocorrelation of the supergranulation pattern for time lags up to six days. This and a longer time series of observations led to the discovery of the wavelike properties of supergranulation (Gizon, Duvall, and Schou, 2003), which suggest that supergranulation is an example of traveling-wave convection. 
The lifetime of the pattern was estimated by Gizon, Duvall, and Schou (2003) to be about two days.

The characterization of the spatial and temporal scales of supergranulation is still a topic of current research. Hart (1956) derived a correlation length of the horizontal flow field of $26 \mathrm{Mm}$, Simon and Leighton (1964) found average cell diameters of $32 \mathrm{Mm}$ and lifetimes of 20 hours. November (1994) found a horizontal length scale of $32 \mathrm{Mm}$, and Hagenaar, Schrijver, and Title (1997), Berrilli et al. (1999), and Srikanth, Singh, and Raju (2000) reported a large number of much smaller cells leading to mean diameters between 10 and $20 \mathrm{Mm}$. Using SOHO/MDI data and local correlation tracking, DeRosa and Toomre (2004) found supergranular cell diameters between 12 and $20 \mathrm{Mm}$. The differences in the observed horizontal scales of supergranulation are mostly due to different methods of data analysis and choices of spatial filters. DeRosa and Toomre (2004) and del Moro et al. (2004) report an exponential distribution of supergranular lifetimes. DeRosa and Toomre (2004) found that $45 \%$ of the tracked supergranules are born or destroyed by a fragmentation or merging event. Another $20 \%$ of supergranules either spawn off or merge with smaller objects during their existence. Only $35 \%$ of objects emerge and dissolve without interaction with adjacent structures.

The distributions of supergranular cell areas presented by Schrijver, Hagenaar, and Title (1997), Hagenaar, Schrijver, and Title (1997), and DeRosa and Toomre (2004) agree well with a Voronoi (skewed, right-asymmetric) distribution of randomly distributed cells, while Berrilli et al. (2004) found good agreement with a hard-sphere close-packing model that deviates from a random placement of cells.

In the present helioseismological study we aim to determine the statistical properties of supergranules, that is, their sizes, flow divergences, and lifetimes, using segmentation algorithms originally developed for granulation. This study differs from previous studies in the sense that we use a very large sample of supergranules. We consider about one order of magnitude more supergranules than Duvall and Gizon (2000) and del Moro et al. (2004).

\section{Data Production}

We used a sequence of full-disk Dopplergrams obtained in 2000 by the Michelson Doppler Imager (MDI Dynamics Program; Scherrer et al., 1995). This time series of Dopplergrams is nearly continuous, has a cadence of one image per minute, and covers 52 days or about two Carrington rotations. The spatial sampling is $0.12^{\circ}$ at disk center. The first step in the analysis was to remap the Dopplergrams by using an azimuthal-equidistant projection and to track the images at the Carrington rotation rate to remove the main component of rotation. The Doppler data were filtered in wavenumber - frequency space to only let through the solar $f$ modes or surface-gravity waves. Solar $f$ modes propagate horizontally and have their maximum kinetic energy at a depth of about $1 \mathrm{Mm}$ below the surface. We then applied the techniques of $f$-mode time - distance helioseismology (Duvall and Gizon, 2000) to obtain a $120^{\circ} \times 120^{\circ}$ map of the horizontal divergence of the horizontal flows in a 1-Mm-deep layer below the solar surface every 12 hours. Using a cross-correlation technique, we measured the time that it takes for $f$ modes to propagate from any spatial point to a concentric annulus of mean radius $15 \mathrm{Mm}$, as well as the travel time in the opposite direction. Since waves are advected by the local flow, the difference in travel time between the ingoing and outgoing waves is essentially proportional to the horizontal divergence of the underlying flow field. The only significant difference between the present analysis and the analysis of Duvall and Gizon (2000) is that we applied a dispersion correction to add $f$ modes of differ- 


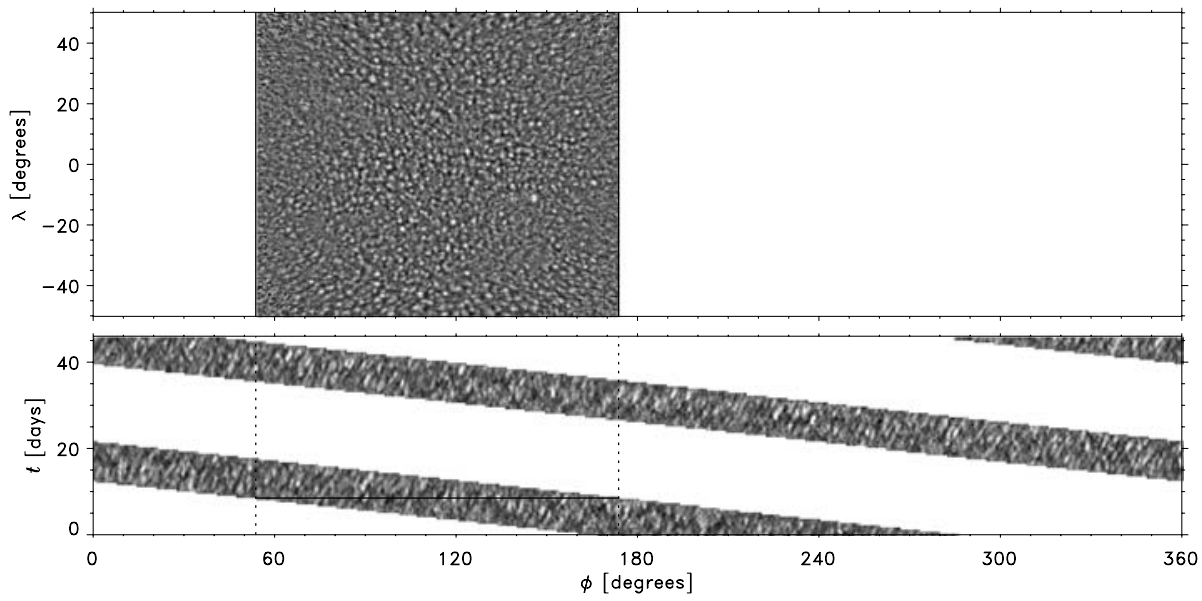

Figure 1 Time series of maps of the horizontal divergence obtained with $f$-mode time-distance helioseismology. The upper panel shows a single map from the series (12-hour average). Bright structures are regions where $\operatorname{div} \boldsymbol{v}_{\mathrm{h}}>0$ (supergranular outflows); dark regions have $\operatorname{div} \boldsymbol{v}_{\mathrm{h}}<0$ (inter-supergranular lanes). The lower panel shows a Carrington longitude - time slice at fixed latitude $\left(\lambda=-2.25^{\circ}\right)$. At each particular time, only one-third $\left(120^{\circ}\right)$ of the entire solar longitude range is observed. The time series analyzed in this paper covers about two Carrington rotations. Supergranules appear as inclined elongated structures in the lower panel as a result of the differential rotation of the supergranular pattern with respect to the Carrington rotation rate.

ent frequencies in phase, before measuring the travel times. The divergence maps were then interpolated onto a longitude - latitude grid and sampled every $0.24^{\circ}$ in both coordinates. The series of 12-hour divergence maps are combined to form a three-dimensional (3D) data cube (Carrington longitude, latitude, and time). In a co-rotating frame, equatorial regions can be followed without interruption for as much as about nine days. Sections through this cube are shown in Figure 1. Supergranules are easy to identify as regions of outflows with a typical spatial scale of $30 \mathrm{Mm}$.

Throughout this paper we refer to the travel-time difference (ingoing minus outgoing travel times) as the "divergence signal": $\operatorname{div} \boldsymbol{v}_{\mathrm{h}}$. Hence, our divergence signal has units of time. A proper conversion of the travel-time signal into the true horizontal divergence of the flow (in units of 1/time) would require a travel-time inversion (e.g., Jackiewicz et al., 2007; Jackiewicz, Gizon, and Birch, 2008), which we are not doing in this paper.

Because of geometrical foreshortening, the divergence maps are of better quality close to disk center than at large heliocentric angles $\theta$. For this reason only data points within $\theta<48^{\circ}$ have been taken into consideration. In addition, a low-pass spatial filter (with a cutoff at spherical harmonic degree $\ell=250$ ) was applied to each individual 12-hour map, to filter out the Fourier components with low signal-to-noise ratios. A filtered divergence map is shown in Figure 2.

Figure 3 shows histograms of the horizontal-divergence values obtained by considering the entire data set for the raw and the spatially filtered data. A slight asymmetry of the distributions is clearly visible, as noticed earlier by Duvall and Gizon (2000). The fraction of areas where $\operatorname{div} \boldsymbol{v}_{\mathrm{h}}>0$ is $46.8 \%$ in the filtered data set. We note that a similar asymmetry is also present in the distributions of Doppler velocities and, more significantly, of continuum intensities measured in "normal" solar granulation (see, e.g., Hirzberger, 2002). 
Figure 2 Divergence map after spatial filtering. Only the points with heliocentric angle $\theta<48^{\circ}$ are kept for analysis. The white square marks the detail displayed in Figure 8.

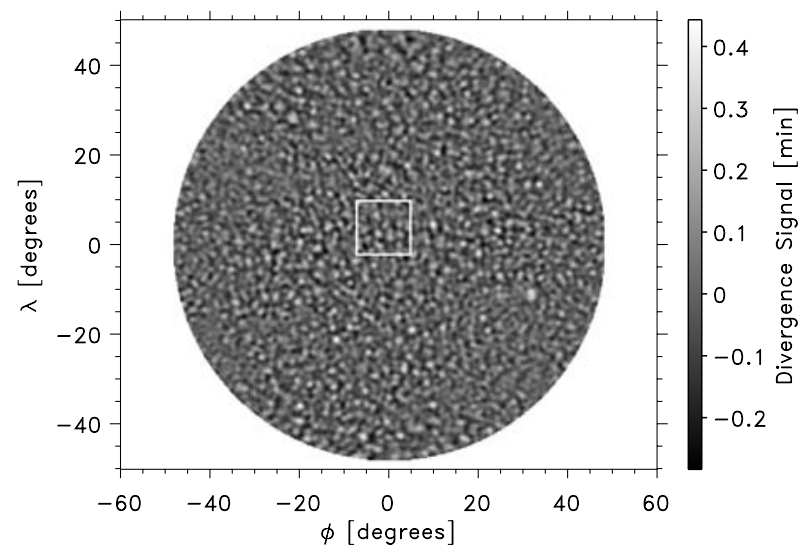

Figure 3 Distribution of the values of the divergence signal [div $\boldsymbol{v}_{\mathrm{h}}$ ] from the entire time series covering two Carrington rotations in the year 2000. The solid curve was obtained from the spatially filtered data; the dotted curve shows raw data.

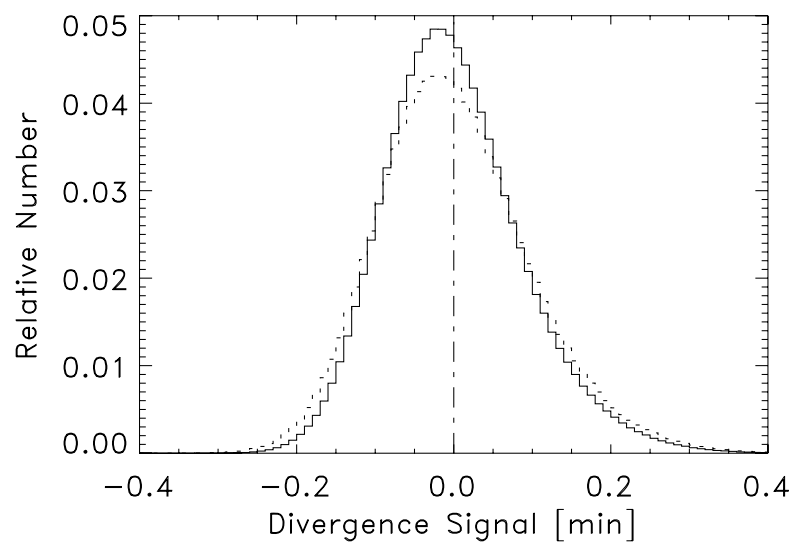

\section{Definition of Supergranular Cells}

After filtering, the obtained divergence maps (Figure 2) show a blotchy structure of distinct areas of strong divergence ( $\operatorname{div} \boldsymbol{v}_{\mathrm{h}}>0$ ) and convergence ( $\operatorname{div} \boldsymbol{v}_{\mathrm{h}}<0$ ) separated by regions containing values close to zero divergence. To analyze the shapes of the individual divergence and convergence centers we apply an image segmentation algorithm to the divergence maps as well as to the convergence maps ( $-\operatorname{div} \boldsymbol{v}_{\mathrm{h}}$ ). The algorithm is based on spatial bandpass filtering and cutting the filtered images at a certain divergence threshold (see Roudier and Muller, 1987). Figure 4 shows the azimuthally averaged power spectrum of one divergence map. The band-pass filter is shown as well. The intensity threshold is set to $\pm 0.8 \sigma$, where $\sigma$ is the standard deviation of the divergence maps. Lower thresholds result in the merging of supergranular cells, which otherwise appear as separated features in Figure 2. Applying higher thresholds leads to holes in the segmentation maps (Figure 5), that is, large regions where no cell is detected by the algorithm. In these regions, the divergence is reduced; however, they are not free from cellular structures. Thus, upon visual inspection, a threshold of $\pm 0.8 \sigma$ would appear to be acceptable. As can be seen from Figure 4 the Fourier filter turns out to be closer to a high-pass than a band-pass filter, similar to the one used by Sánchez Cuberes et al. (2000). However, since structures smaller than $\ell=250$ have been already filtered out during the preprocessing stage, the band-pass character is retained. 
Figure 4 Spatial power spectrum of a divergence map (thick solid line). The dotted line shows the low-pass filter and the dash-dotted line shows the band-pass filter applied to the data for the segmentation of the divergence signal. The thin solid curve shows the power spectrum of the image after applying the two filters.

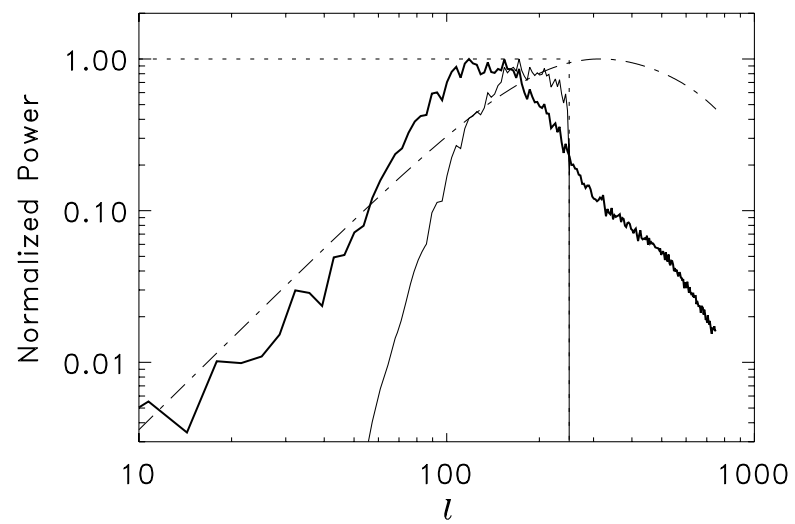

Even after filtering out structures smaller than $\ell=250$ the segmentation process produces structures with much smaller sizes if the applied threshold is only slightly lower than the maximum divergences of the segmented structures. Therefore, structures with areas smaller than four pixels were removed from the segmented maps.

Examples of segmented divergence and convergence maps are shown in Figure 5. The resulting divergence centers (see Figure 5(a)) are mostly small structures with regular shapes, whereas the convergence centers (Figure 5(b)) seem to be larger and much more irregularly shaped. This visual impression is quantified by the distributions of areas shown in Figure 6. The higher complexity of shapes of convergence centers can also be confirmed by computing the fractal dimensions $D_{f}$ from the perimeter-area relation (see Mandelbrot, 1977). We found values of $D_{f}=1.56$ for the divergence areas and $D_{f}=1.76$ for the convergence areas. Thus, supergranulation is indeed characterized by regular cells with $\operatorname{div} \boldsymbol{v}_{\mathrm{h}}>0$, surrounded by "inter-supergranular lanes" with $\operatorname{div} \boldsymbol{v}_{\mathrm{h}}<0$.

Following this characterization, a "supergranulation cell" can be defined as an area of positive divergence surrounded by a part of the inter-supergranular lanes. Therefore, we extended each divergence area obtained by the Fourier segmentation toward its surrounding local minima of $\operatorname{div} \boldsymbol{v}_{\mathrm{h}}$. The local minima were defined as pixels that have a smaller divergence value than the eight surrounding pixels in a three $\times$ three square pixel box. The areas of the previously deleted structures smaller than four pixels are, thus, merged with that of the nearest larger supergranule. This dilation algorithm is similar to the "watershed basin segmentation" used in DeRosa and Toomre (2004). An example of such a dilated map is shown in Figure 7. From these maps a sample of more than $1.19 \times 10^{5}$ supergranular cells has been extracted. The entire segmentation process is illustrated in Figure 8 on a detail of the divergence map displayed in Figure 2.

To test the performance of this procedure, mean divergence values averaged over the resulting cells were calculated. If the definition of the cells is reasonable these average divergences should be close to zero. A corresponding histogram is shown in Figure 9. This resulting distribution is well fitted by a Gaussian centered at zero divergence and with a standard deviation of $\sigma=0.022$. For comparison, histograms of mean divergences averaged over divergence and convergence centers obtained after the Fourier segmentation are overplotted in Figure 9. These distributions are significantly broader than the one calculated from the cells. The two curves meet at $\operatorname{div} \boldsymbol{v}_{\mathrm{h}}=0$, which means that Fourier segmentation indeed produces reasonably defined structures. Both curves show a significant skewness. However, the skewness is more pronounced for the divergence than for the convergence centers, which 
Figure 5 Image segmentation based on Fourier band-pass filtering applied to the image displayed in Figure 2 (a) and to its negative (b). The white areas in panel (a) represent detected supergranular divergence centers; gray areas in panel (b) are inter-supergranular convergence centers.
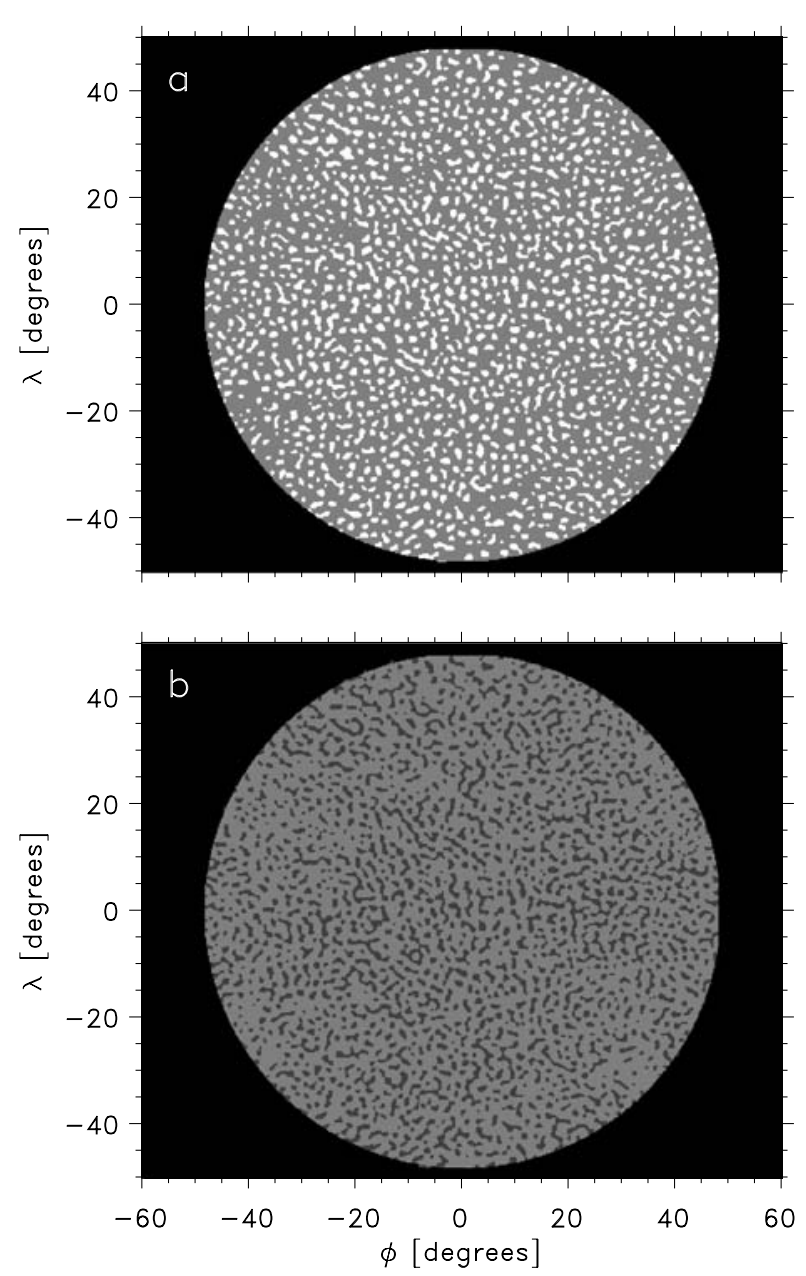

is consistent with the distribution of divergence values in nonsegmented data ( $c f$. Figure 3) and granular intensities and velocities (see Hirzberger, 2002). This result indicates that there is a similarity between supergranulation and granulation.

\section{Statistical Analysis of Cell Structure}

After applying image segmentation, we can assign several parameters to each detected supergranular cell. Figure 10 shows distributions of cell areas $[A]$ and maximum divergence values $\left[\xi \equiv\left(\operatorname{div} v_{\mathrm{h}}\right)_{\max }\right]$ in the cells. The cell area varies between 160 and about $2000 \mathrm{Mm}^{2}$, which corresponds to an effective cell diameter, defined as $d=\sqrt{4 A / \pi}$, between 14 and $50 \mathrm{Mm}$. The median cell area is $650 \mathrm{Mm}^{2}$ and the median cell diameter is $28.8 \mathrm{Mm}$. These latter numbers were obtained by considering the entire sample of segmented supergranular cells.

We note that the shape of the histograms of cell areas depends on the parameters of the segmentation procedure and, in particular, on the filtering. The cutoff value of $0.8 \sigma$ 
Figure 6 Histograms of areas of divergence centers (solid) and convergence centers (dotted) obtained by applying the image segmentation procedure based on Fourier pass-band filtering.

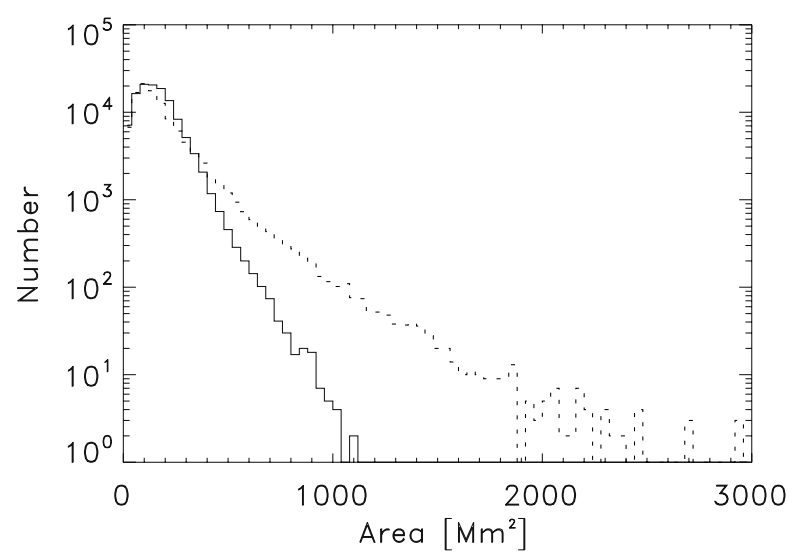

Figure 7 Dilation of detected supergranular areas of the map shown in Figures 2 and 5 toward their surrounding local divergence minima, leading to the delimitation of the supergranular cells (blue mesh). The red areas represent divergence centers detected by the segmentation algorithm.

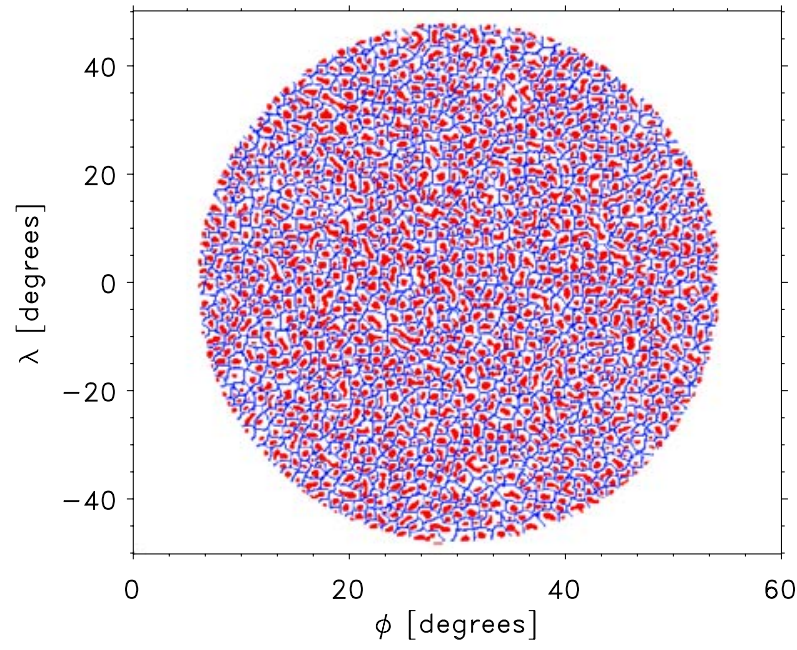

(defined earlier) provides the maximum number of cells. Lowering this threshold results in merging structures, which reduces the number of detected cells and narrows the width of the histograms since the very small cells are less numerous. If the threshold is increased, then the very faint structures are ignored, which also reduces the total number of cells, whereas the histograms get broader. Smaller scales are present, but the regions where no structures are detected become filled by expanding the adjacent supergranules. This leads to several unrealistically large cells.

As can be seen in Figure 10 the distribution of cell sizes changes slightly with heliographic latitude $[\lambda]$. A monotonic decrease of the average cell size from the Equator toward higher latitudes can be detected. A similar trend, although weaker, can be seen for $\xi$ shown in the lower left panel of Figure 10. Since this latter parameter only weakly depends on the definition procedure of a supergranular cell, this behavior is probably not caused by the segmentation algorithm. This conclusion is strengthened by the results shown in the right panels of Figure 10. Here the variation of the area and divergence histograms with heliocentric angle $\theta$ is plotted. The obtained trend is even more significant than the variation with $\lambda$. 
Figure 8 Illustration of the segmentation procedure: The lower left panel shows a detail $\left(6^{\circ} \times 6^{\circ}\right)$ of the filtered divergence map displayed in Figure 2; in the upper panels the corresponding segmented divergence (left) and convergence (right) centers (see Figure 5) are shown; in the lower right panel the divergence centers and their dilations to supergranular cells (see Figure 7) are depicted.
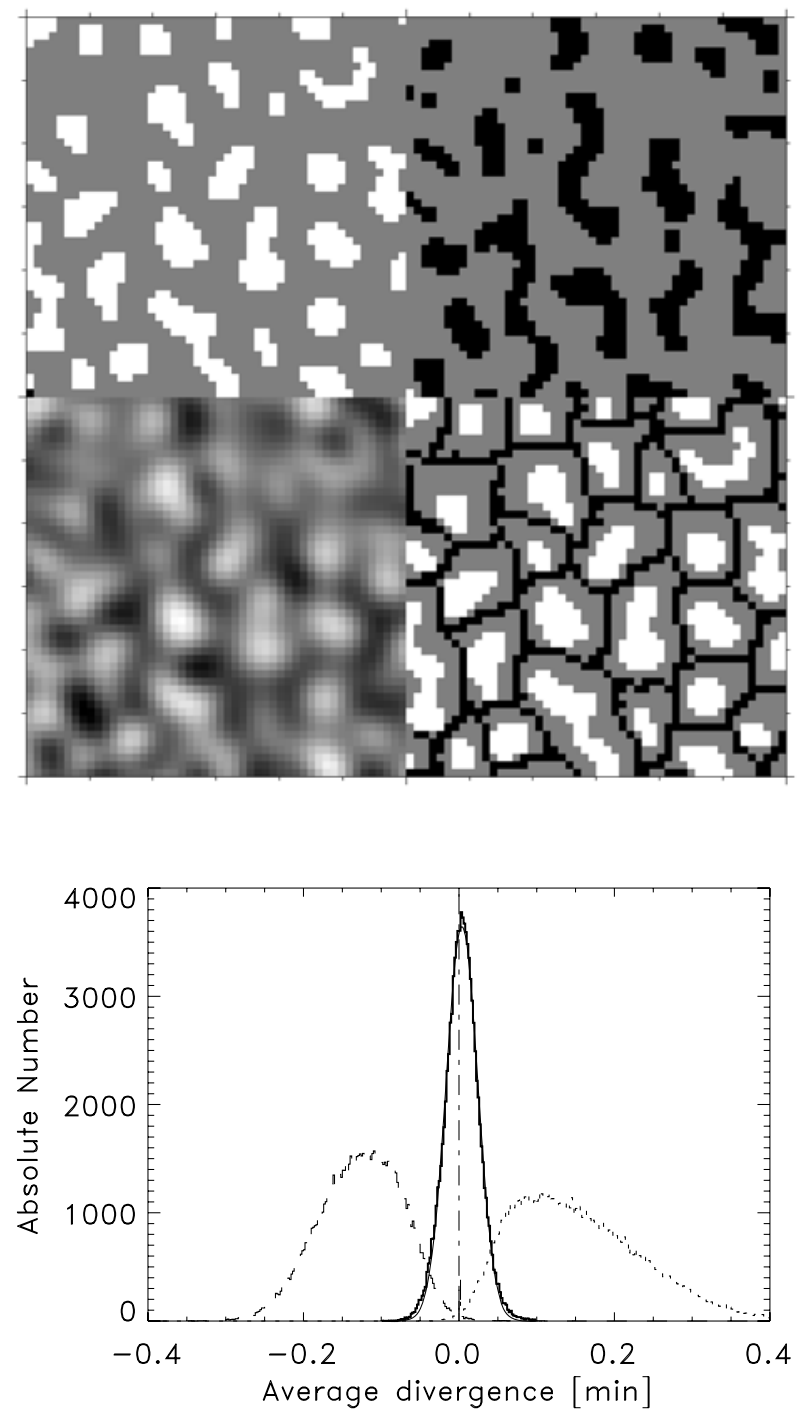

Figure 9 Histograms of average divergence for supergranular cells (thick solid line), for divergence centers (dotted), and for convergence centers (dashed). The (barely visible) thin solid line represents a Gaussian fitted to the cell histogram.

Consequently, it must be concluded that the observed latitudinal variation of supergranular size and divergence is not of solar origin only but is likely to be an intrinsic effect of the data processing using helioseismology techniques.

The shapes of the supergranular size distributions are characterized by a significant asymmetry, similar to the findings of, for example, Hagenaar, Schrijver, and Title (1997) and DeRosa and Toomre (2004). Compared to the resulting cell sizes reported in del Moro et al. (2004) the distribution shows a significantly steeper drop from the maximum toward small cells and also a slight shift of the location of the maximum value toward smaller cells. (We find $A=575 \mathrm{Mm}^{2}$ and $d=27.1 \mathrm{Mm}$, respectively, compared with $d=33 \mathrm{Mm}$ reported in del Moro et al. (2004)). Another conspicuous feature of the histograms is the appearance of a minimum cell area of $158 \mathrm{Mm}^{2}$, which arises because of the applied minimum structure 

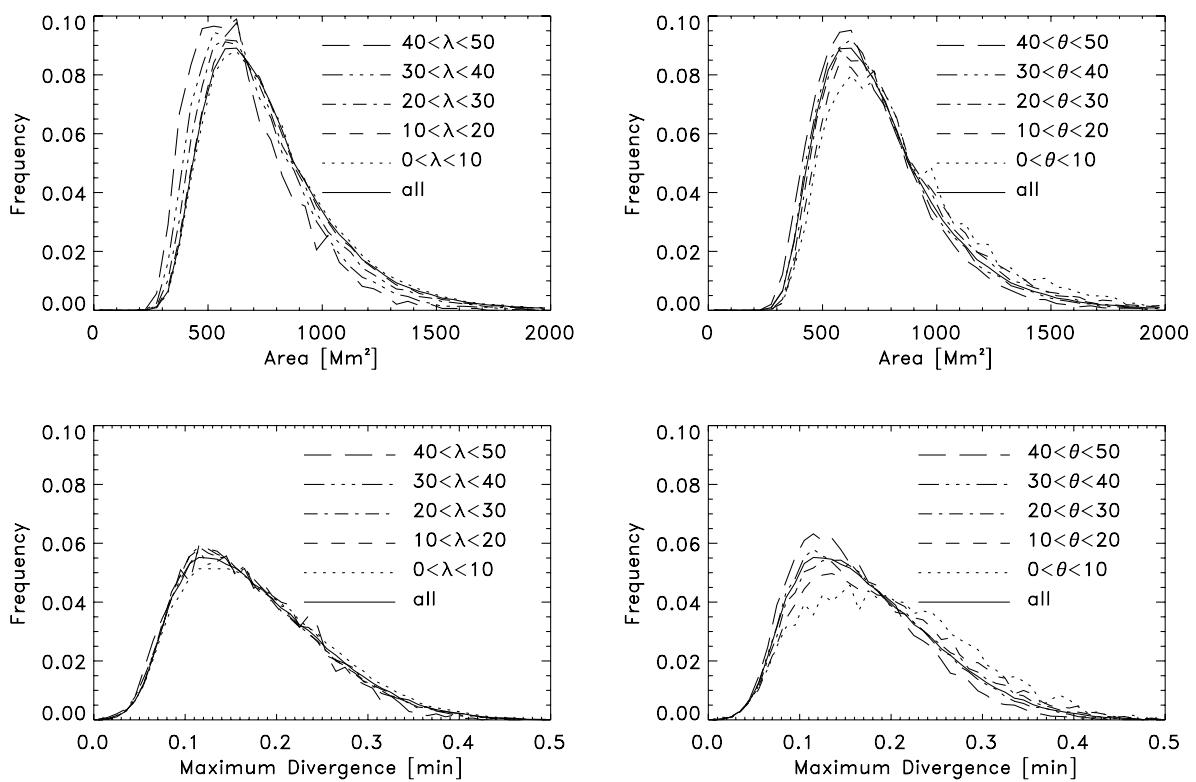

Figure 10 Histograms of areas $A$ (upper panels) and maximum divergences $\xi$ (lower panels) of supergranular cells obtained in different regions on the solar disk. The curves in the left panels have been obtained by considering stripes of different latitudes $\lambda$; the curves in the right panels are computed by changing heliocentric angle $\theta$.

size in the Fourier segmentation. This fact, however, cannot explain the differences compared to the results of del Moro et al. (2004) since there a similar minimum size condition was applied. Another possibility may be the different (presumably larger) noise level in the data used by del Moro et al. (2004). Residual noise leads to a decrease of the obtained cell sizes. For instance, if the low-pass filter (allowing only structures larger than $\ell=250$ ) is not applied to our data the median value of the cell areas decreases from 650.6 to $603.0 \mathrm{Mm}^{2}$. However, the obtained shapes of the histograms do not change from right-asymmetric to left-asymmetric as found in del Moro et al. (2004).

The close similarity of shapes of the area and divergence distributions visible in Figure 10 suggests a close relation of both parameters. Owing to the large number of structures in our sample, a 2D histogram is more appropriate for showing this relation than a simple scatter plot (see Figure 11). In the range $300<A<700 \mathrm{Mm}^{2}$ a linear relation between the two parameters can be observed. Toward larger cells, the scatter of the distribution increases and the area-divergence relation becomes ambiguous. A close relation between size and flow divergence of supergranules is not surprising and was also reported by Meunier et al. (2007).

\section{Temporal Evolution}

Once supergranular cells have been identified in each image, it becomes possible to follow the temporal evolution of individual structures. We applied a feature-tracking code originally developed to track granules (Hirzberger et al., 1999). A modified version of the code 
Figure 11 Two-dimensional histogram of maximum divergence versus supergranular cell area.

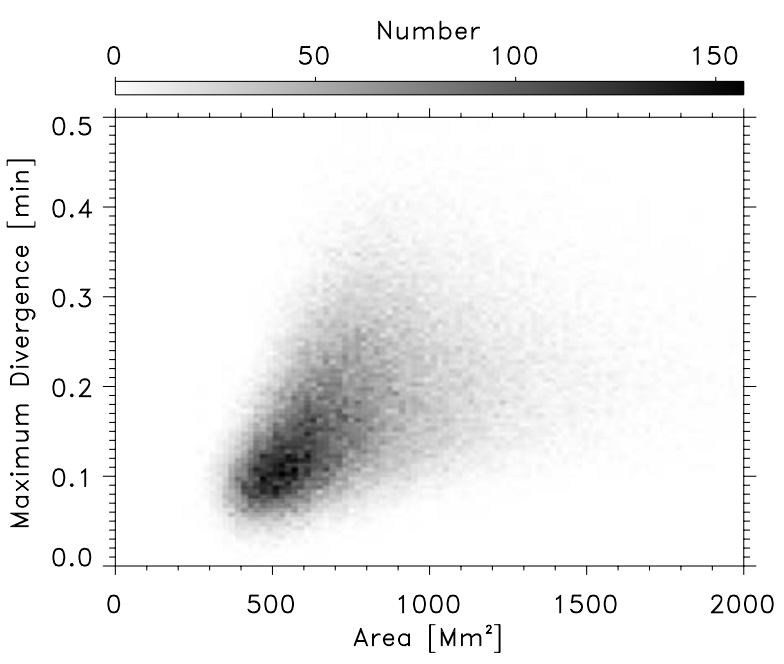

was also applied to mesogranules (Leitzinger et al., 2005). This code tracks a certain feature forward and backward in time until a stopping condition is reached, where the tracking is terminated. The length of time between the two termination points is the structure's lifetime $[T]$. In the present version of the code, the lifetime history of a structure was terminated if $A(t)>2 A(0)$ or if $A(t)<A(0) / 2$, where $A(0)$ is the area of the cell when the tracking was started and $A(t)$ is its area at time $t$. The tracking was also terminated if the barycenter of a structure seems to jump more than three pixels between subsequent images. However, before applying this criterion, the structures where shifted by the proper motions caused by the mean rotation at the appropriate latitude. This second criterion avoids connecting the lifetime history of a feature with that of a neighboring one after the tracked feature vanishes. Finally, the resulting lifetime histories were smoothed by applying a parabolic fit to $A(t)$ and removing those points that are outside $\pm 1.5 \sigma$ of the fit from the histories, where $\sigma$ denotes the standard deviation of the fit from the data points. The obtained histories were cut when more than two subsequent data points were removed by the fit. If the stopping conditions were used in a restrictive way, residual noise would lead to an artificial shortening of the lifetime histories. The fitting procedure was implemented to mitigate the stopping conditions and to reconsider the performance of the tracking procedure. Redundantly tracked features and those histories that reach the boundaries of the data cube were deleted from the sample of evolutional histories. However, keeping only those histories that describe the entire lifetime of a supergranule cell from its birth to its death causes some bias to the obtained results since short-lived structures are favored. Altogether a sample of 3529 lifetime histories was obtained by applying this procedure. This number is small compared to the number of detected cells. This is because only a small fraction of histories is not truncated by the image borders or by rotating out of the field of view.

A second method to characterize the temporal evolution of supergranular features is to apply $3 \mathrm{D}$ version of the Fourier-based segmentation to the data. The pass-band filter (see Figure 4) was extended to three dimensions. We assumed ergodic conditions (i.e., the shapes of the supergranules are assumed to be statistically uniform in $(\phi, \lambda, t)$-space). The time dimension was treated as a third spatial dimension and the filter described in Roudier and Muller (1987) was applied to the 3D data cube as was done before to the 
Figure 12 The upper panel shows histograms of supergranular lifetimes. Asterisks show the results from the feature-tracking method and diamonds from the 3D Fourier segmentation. The solid line represents an exponential fit to the feature-tracking data; the dotted curve shows a fit to the $3 \mathrm{D}$ segmented data. Also shown are the lifetime dependence of areas (middle panel) and divergences (lower panel) for both segmentation methods. Red solid lines give the mean $A$ and $\xi$ from feature tracking; red dashed lines give the maximum $A$ and $\xi$ from feature tracking; blue solid lines give the mean $A$ and $\xi$ from 3D Fourier segmentation; blue dashed lines give the maximum $A$ and $\xi$ from 3D segmentation. Error bars denote standard deviations of the mean $(\sigma / \sqrt{N})$.
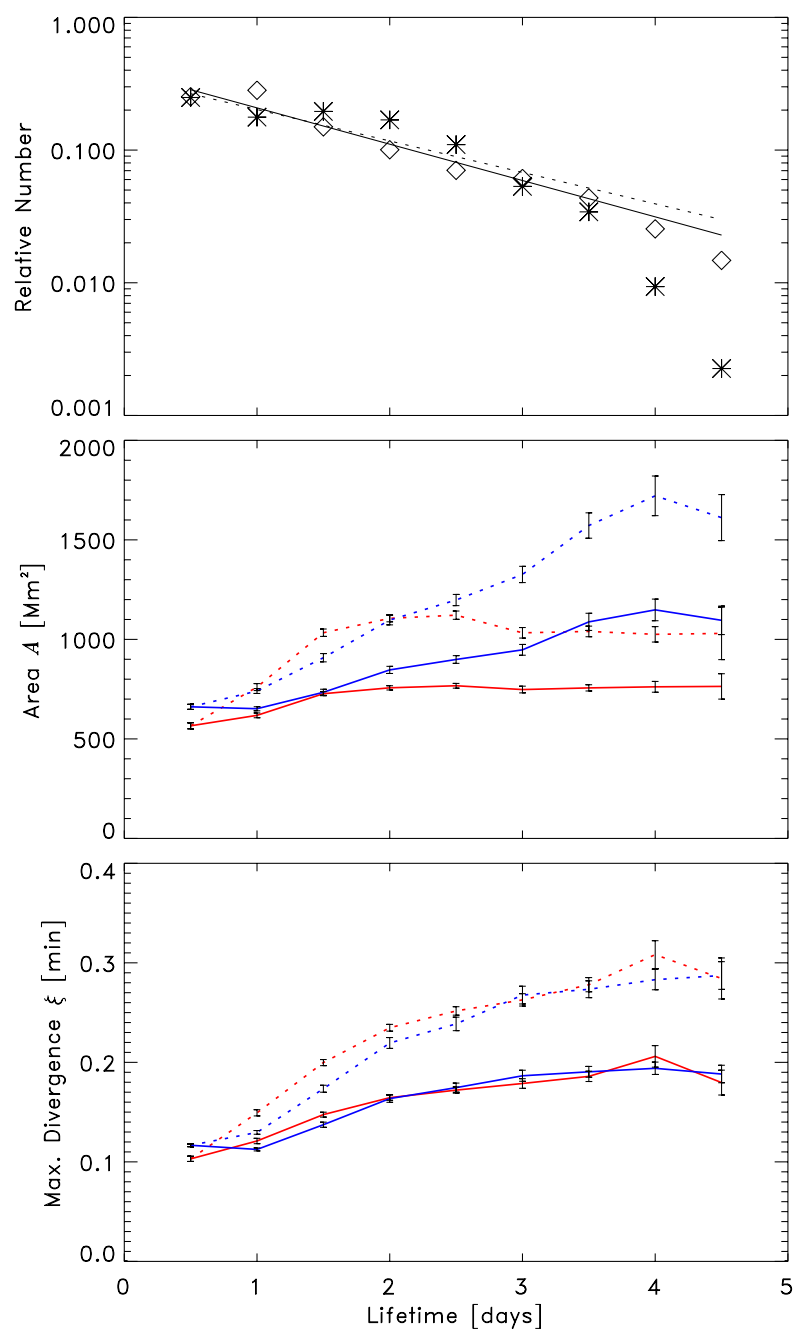

$2 \mathrm{D}$ divergence maps. After Fourier filtering and cutting at a level of $\pm 0.8 \sigma$ above the mean (see Section 3), the lifetimes of resulting structures are simply given by their extent along the time axis. To retrieve supergranular cell areas, the data cube was then sliced into single images and each feature was extended toward the surrounding local divergence minima. Again all histories that reach the boundaries of the data cube have been deleted. In addition, those structures that are smaller than eight pixels in $(\phi, \lambda, t)$-space were deleted. The supergranular areas computed by using 3D segmentation are almost identical to those found with the $2 \mathrm{D}$ version of the code. However, structures that have small extents, particularly in time, are deleted by the code. This leads to a significant reduction of the number of structures in the sliced images and, therefore, to an increase of the mean cell sizes when dilating the areas to the local divergence minima. Altogether, from the 3D image segmentation a sample of 4977 lifetime histories was obtained. 
The inferred evolution parameters depend considerably on the segmentation and tracking methods (see, e.g., Alissandrakis, Dialetis, and Tsiropoula, 1987, for a comparison of lifetime estimation procedures). The lifetime distributions (Figure 12) show a decrease of the relative frequency of cells toward longer lifetimes. The lifetime distribution obtained from the $3 \mathrm{D}$ segmentation may be modeled with an exponential distribution of the form $N(T)=N(0) \mathrm{e}^{-T / \tau}$, where $\tau$ is the mean lifetime. We find $\tau=1.59$ days, which is somewhat longer than the classical value of one day (Leighton, Noyes, and Simon, 1962) and longer than the value $\tau=0.92$ days stated by del Moro et al. (2004). The standard deviation of $\tau$ is $\sigma_{\tau}=0.74$ days. Note that for $T$ longer than four days the effect of deleting histories that are truncated by the data boundaries leads to a deviation from the exponential form. If the first data point at $T=0.5$ days is omitted from the fit, then the resulting distribution looks steeper and $\tau$ becomes 1.12 days (with a standard deviation $\sigma_{\tau}=0.59$ days). Thus the classical lifetime of one day lies within the uncertainty of the present result.

The histogram obtained from the feature-tracking analysis shows stronger deviations from an exponential function than the Fourier-based histogram. A significant excess of structures at lifetimes around two days can be observed. Both short-lived ( $T<1.5$ days) and long-lived ( $T>3.5$ days) supergranules are comparatively less frequent than in the sample obtained from the 3D segmentation. An exponential fit gives $\tau=1.83$ days with a standard deviation of $\sigma_{\tau}=0.89$ days.

The middle and lower panels of Figure 12 show cell areas and maximum divergences (i.e., the largest divergence within a supergranule at any given time) averaged over each lifetime history and, additionally, averaged over each bin of the histograms. The 3D segmentation shows a clear trend for larger and more divergent supergranular cells to live longer. The mean area even seems to be linearly related to the lifetime. The feature tracking reveals such a trend only for short-lived cells, while displaying a very similar trend to the $3 \mathrm{D}$ segmentation for the maximum divergence. Supergranules with lifetimes $T \geq 2$ days have constant mean areas of $750 \mathrm{Mm}^{2}$. This latter behavior is in almost perfect agreement with the lifetime - area relation presented in del Moro et al. (2004). The lifetime-area and the lifetime-divergence relations do not change significantly if the maximum area and divergence of each lifetime history is considered instead of using temporal means of each history (dashed and dash-dotted lines in Figure 12).

Typical time evolution histories of parameters $A$ and $\xi$ are shown in Figures 13 and 14 . The curves represent averages of all histories included in a certain bin of the lifetime histograms derived from both 3D Fourier segmentation and feature tracking (see Figure 12). In the lifetime range $1 \leq T \leq 4.5$ days, the evolution shows typically convex shapes: A supergranular cell and its divergence are small when it is "born." After that, area and divergence increase until roughly half the lifetime is reached. After that, both parameters are decreasing again until the cell "dies." Very approximately, the time dependence of supergranule area and maximum divergence is quadratic. Within the achievable accuracy it is difficult to say whether there is a general asymmetry in the sense that the supergranules reach their maximum size in the first or the second half of their lifetime. This behavior is particularly well pronounced for the mean histories obtained from the 3D segmentation and more noisy in the feature-tracking results. Moreover, as already mentioned, the mean cell areas are significantly larger in the $3 \mathrm{D}$ than in the $2 \mathrm{D}$ segmentation. The mean evolution histories of the longest living supergranular cells do not seem to follow a clear trend, probably because of the low number of histories in the corresponding samples. The retrieved convex lifetime histories are considerably different from the histories of granulation cells (see, e.g., Hirzberger et al., 1999). They reach their maximum size at the end of their lifetimes (i.e., the largest fraction of granular cells increase in size until they split into two or more fragments). 

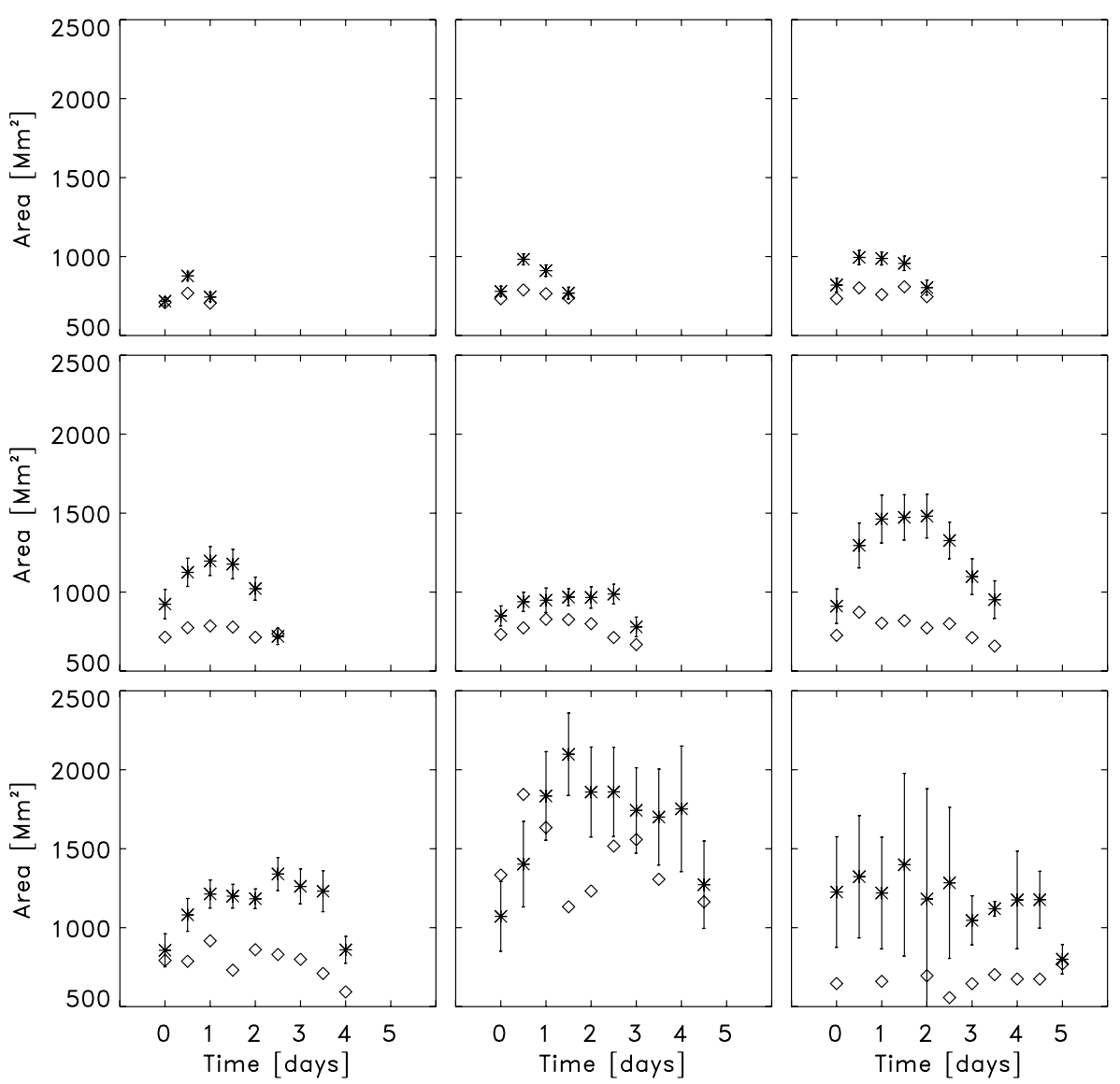

Figure 13 Mean area evolution of supergranules with different lifetimes. Each panel shows mean evolutions determined by averaging $A$ over all histories in a single bin of the 3D segmentation (asterisks) and feature-tracking (diamonds) histograms shown in Figure 12. The error bars denote standard deviations of the mean.

\section{Pair Correlation Functions}

Important aspects of the supergranulation phenomenon are not only given by the properties of individual cells. For a detailed understanding of supergranulation the interaction between neighboring cells and, particularly, the arrangement of cells within a certain region might be highly instructive.

A useful tool to analyze the arrangement of structures in a given region is the so-called pair correlation function (PCF), which represents the probability of finding a supergranule located at a distance $r$ from another supergranule. A comparison of the PCFs of granulation and supergranulation has already been performed by Berrilli et al. (2004). In the present work the position of a supergranular cell is represented by its barycenter, which is defined by its centroid weighted by its divergence. For a homogeneous and isotropic distribution of 

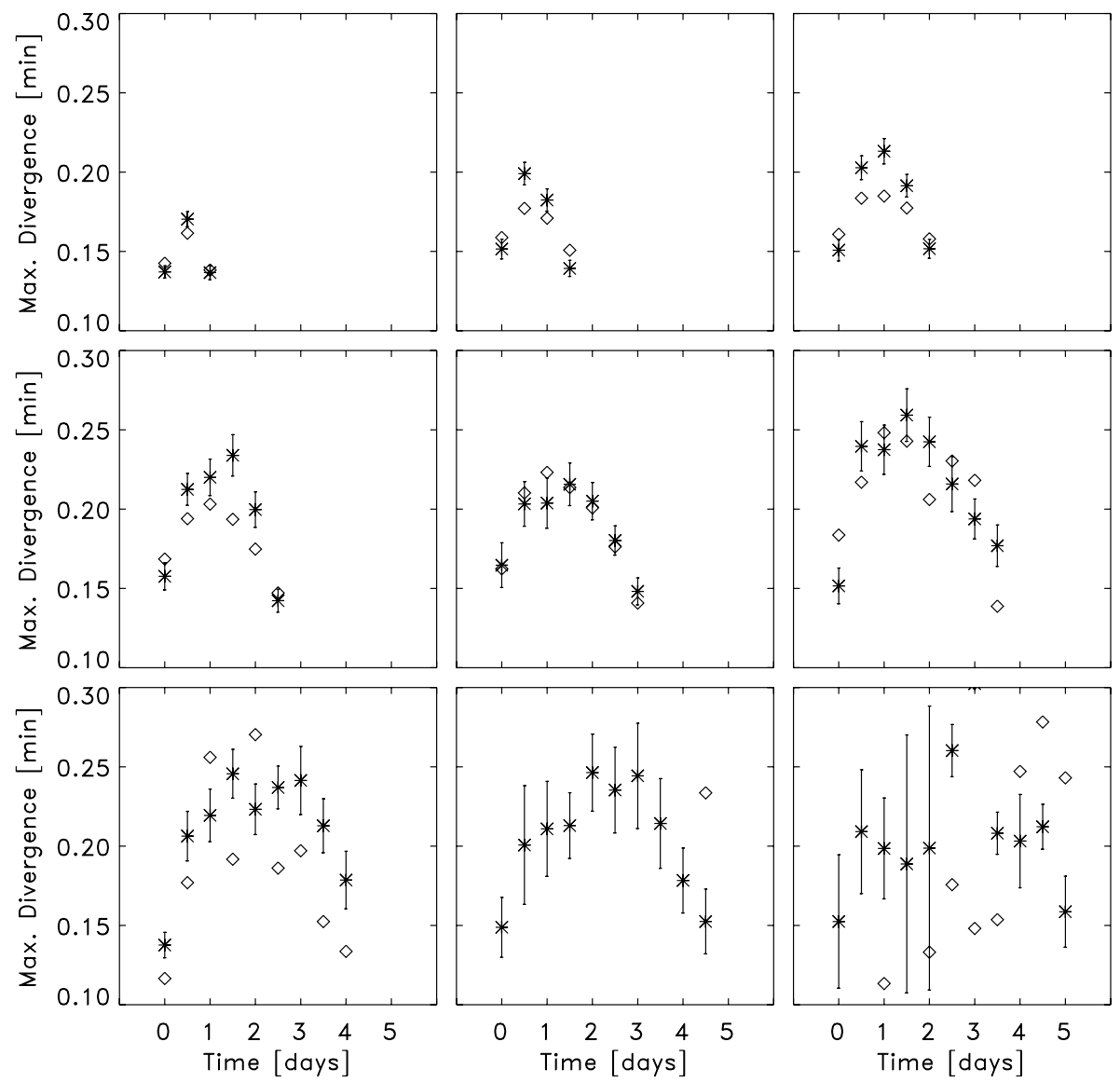

Figure 14 Mean evolution of supergranules with different lifetimes. Each panel shows mean evolutions determined by averaging $\xi$ over all histories in a single bin of the 3D segmentation (asterisks) and feature-tracking (diamonds) histograms shown in Figure 12. The error bars denote standard deviations of the mean.

structures, the PCF $[g(r)]$ is defined as

$$
g(r)=\frac{\rho(r)}{\eta^{2}},
$$

where $\rho(r)$ is the product density, defined in the following. The function $g$ describes the probability of finding exactly one structure in each of the two infinitesimal small circles at positions $\boldsymbol{x}^{\prime}$ and $\boldsymbol{x}^{\prime \prime}$, separated by the distance $r$, and $\eta$ is an intensity measure (i.e., the mean number of structures in an area of size unity). The PCF depends on the product density (see Stoyan and Stoyan, 1994):

$$
\rho(r)=\frac{1}{2 \pi r} \sum_{i=1}^{n} \sum_{\substack{j=1 \\ j \neq i}}^{n} \frac{K_{h}\left(r-\left\|\boldsymbol{x}_{j}-\boldsymbol{x}_{i}\right\|\right)}{A\left(W_{j} \cap W_{i}\right)},
$$



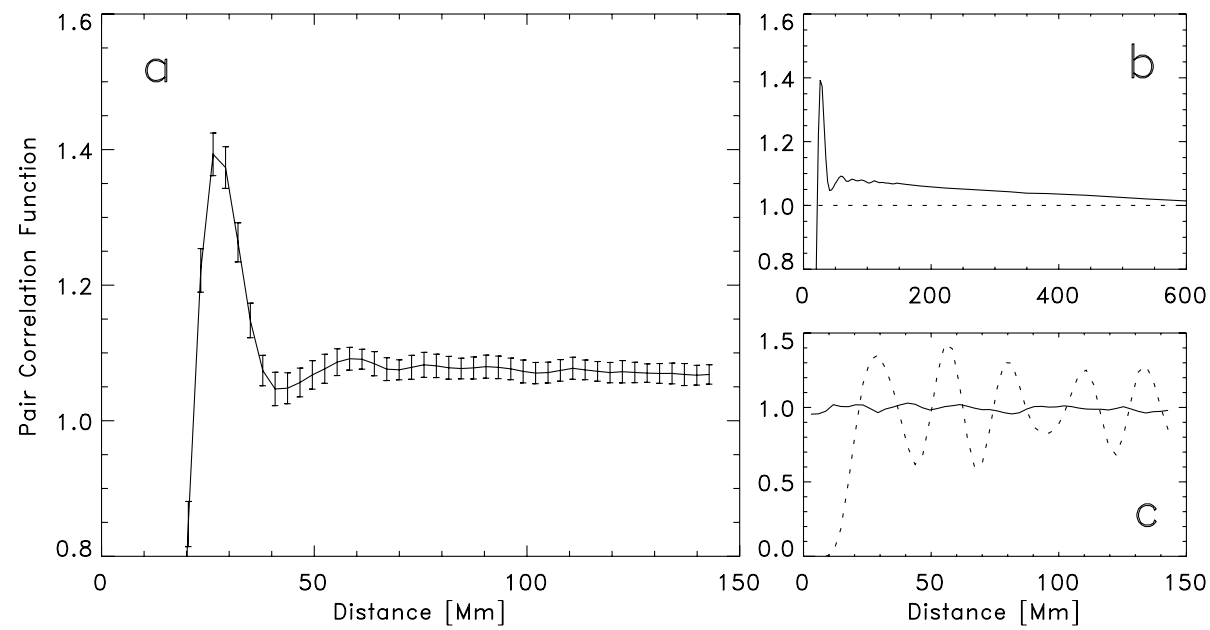

Figure 15 (a) Mean PCF obtained by averaging the PCFs of each 2D segmented divergence map. Error bars denote standard deviations. (b) The same PCF for distances up to $r=600 \mathrm{Mm}$. (c) For reference, the PCFs of a random configuration (solid) and a randomly disordered regular cell pattern (dotted) are shown.

where the Epanečnikov kernel is defined by

$$
K_{h}(r)= \begin{cases}\frac{3}{4 h}\left(1-r^{2} / h^{2}\right) & \text { if }-h \leq r \leq h \\ 0 & \text { else. }\end{cases}
$$

In this kernel, the "bandwidth" $h$ is a free parameter that has to be defined externally. In the present study a bandwidth of $h=2$ pixels is used. The summation in Equation (2) runs over all $n$ structures of the sample that are located at positions $\boldsymbol{x}_{i}$ and $\boldsymbol{x}_{j}$, respectively. The area $A\left(W_{j} \cap W_{i}\right)$ is given by the intersection of maps shifted once by $\boldsymbol{x}_{i}$ and once by $\boldsymbol{x}_{j}$. This form avoids bias from structures located close to the borders of the considered region where the distribution of structures within a circle with radius $r$ is not homogeneous since the circle is divided by the border. The normalization of $g$ is fixed by the factor $\eta^{2}=n(n-1) / A(W)$, where $A(W)$ is the total area.

For each 2D segmented divergence map, a PCF was calculated. A mean PCF derived from averaging the functions calculated for each map is displayed in Figure 15. The plot shows a conspicuous peak at $r=28 \mathrm{Mm}$ and a less distinct minimum at $r=43 \mathrm{Mm}$. This result gives strong evidence that a preferred distance between adjacent supergranular cells is $28 \mathrm{Mm}$ and a slight tendency that distances of $43 \mathrm{Mm}$ are rather unlikely. In contrast to the results of Berrilli et al. (2004), a clear second maximum is not visible and even the first minimum is only weakly pronounced (and above unity). The position of the maximum of the PCF corresponds well to the diameter of the preferred cell size (see Figure 10). The standard deviation of the peak position, obtained from polynomial fits to the individual PCFs, is $\sigma_{\max }=0.55 \mathrm{Mm}$ and that of the minimum position is $\sigma_{\min }=1.59 \mathrm{Mm}$. The shape of the obtained PCF - only one conspicuous maximum and a weakly pronounced minimum - is typical for so-called soft-core fields (see Stoyan and Stoyan, 1994). Soft-core fields are good approximations for fields of nonoverlapping circles with variable diameters. This is unlike 

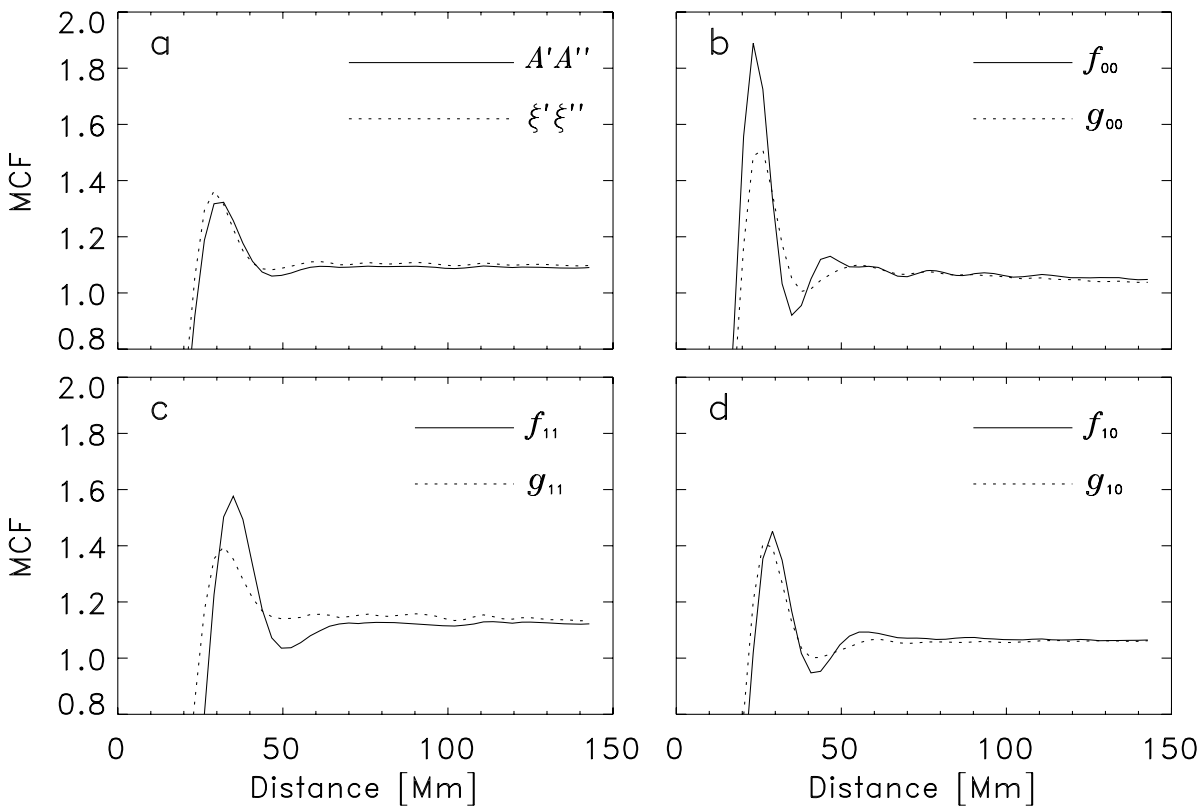

Figure 16 Mean MCFs obtained by averaging the MCFs of each 2D segmented divergence map. The four panels represent different test functions.

the results of Berrilli et al. (2004), who have assumed a hard-sphere close-packing model (i.e., close-packed circles of uniform diameters).

The definition of $g(r)$ (Equation (1)) implies that it tends to one as $r$ tends to infinity. Figure 15(b) shows a convergence to unity for large distances $(r \gtrsim 600 \mathrm{Mm})$. For comparison, Figure 15(c) shows PCFs for a random distribution of cells (with a mean distance of $28 \mathrm{Mm}$ ) and for a regular 28-Mm grid for which the cell positions were randomly disordered (up to a maximum distance of $11.66 \mathrm{Mm}$, i.e., four pixels in both the $x$ - and $y$-directions). The distance needed to converge to unity is called the (correlation) range of the PCF. The long range for the supergranular cell positions indicates some kind of long-distance interaction of the cells.

The concept of PCFs can be extended by attributing a certain property (i.e., a mark) to each structure in the map. If the marks $m^{\prime}$ and $m^{\prime \prime}$ are attributed to the positions $\boldsymbol{x}^{\prime}$ and $\boldsymbol{x}^{\prime \prime}$, separated by a distance $r$, then the so-called $f$-product density $\left[\rho_{f}(r)\right]$ is equal to a nonnegative test function $f\left(m^{\prime}, m^{\prime \prime}\right)$ if exactly one structure is located in infinitesimally small circles around $\boldsymbol{x}^{\prime}$ and $\boldsymbol{x}^{\prime \prime}$. If this condition is not fulfilled then $\rho_{f}(r)=0$. With the $f$-product density the so-called mark correlation function (MCF), $k_{f}(r)$, can be defined as

$$
k_{f}(r)=\frac{\varkappa_{f}(r)}{\varkappa_{f}(\infty)},
$$

where

$$
\varkappa_{f}(r)=\frac{\rho_{f}(r)}{\rho(r)} .
$$


In practice, the $f$-product density and, hence, the MCF can be estimated (see Stoyan and Stoyan, 1994) with

$$
\rho_{f}(r)=\frac{1}{2 \pi r} \sum_{i=1}^{n} \sum_{\substack{j=1 \\(j \neq i)}}^{n} \frac{f\left(m_{i}^{\prime}, m_{j}^{\prime \prime}\right) K_{h}\left(r-\left\|\boldsymbol{x}_{j}-\boldsymbol{x}_{i}\right\|\right)}{A\left(W_{j} \cap W_{i}\right)} .
$$

The shape of the test function is a free parameter. In the present work we use $f\left(m^{\prime}, m^{\prime \prime}\right)=$ $m^{\prime} m^{\prime \prime}$. The quantities $m^{\prime}$ and $m^{\prime \prime}$ can be either the area [A] or the maximum divergence [ $\left.\xi\right]$ of a supergranule. The MCF is large if at a certain distance $r$ the function $f$ (e.g., the product $A^{\prime} A^{\prime \prime}$ or the product $\left.\xi^{\prime} \xi^{\prime \prime}\right)$ is large. In Figure 16 the mean MCFs are displayed for various choices of $m$ and $m^{\prime}$. Both MCFs show a distinct maximum for distances slightly larger than $30 \mathrm{Mm}$ and a shallow minimum in the range between 40 and $50 \mathrm{Mm}$. At larger distances no conspicuous features are visible. The MCF for $\xi$ seems to be slightly shifted toward smaller distances than that for $A$ ( $c f$. Figure 16(a)). This shows, in correspondence to the results shown in Figure 11, that for large supergranules a stringent linear relation between cell sizes and maximum divergences is not given. From the shapes of these MCFs it might be concluded that the positions of large and/or divergent supergranules are not different from that of the overall sample.

Instead of marking the structures with true areas and divergence values, both parameters can be discretized. If $\bar{A}$ and $\bar{\xi}$ are the mean values of the supergranular cell areas and maximum divergences, than the following discretized values can be defined:

$$
A_{0}=\left\{\begin{array}{ll}
1 & \text { if } A \leq \bar{A}, \\
0 & \text { if } A>\bar{A}
\end{array} \quad \text { and } \quad A_{1}= \begin{cases}1 & \text { if } A \geq \bar{A}, \\
0 & \text { if } A<\bar{A}\end{cases}\right.
$$

and

$$
\xi_{0}=\left\{\begin{array}{ll}
1 & \text { if } \xi \leq \bar{\xi}, \\
0 & \text { if } \xi>\bar{\xi}
\end{array} \quad \text { and } \quad \xi_{1}= \begin{cases}1 & \text { if } \xi \geq \bar{\xi} \\
0 & \text { if } \xi<\bar{\xi}\end{cases}\right.
$$

Using these definitions, we can consider the following test functions:

$$
f_{00}=A_{0}^{\prime} A_{0}^{\prime \prime}, \quad f_{11}=A_{1}^{\prime} A_{1}^{\prime \prime}, \quad f_{10}=A_{1}^{\prime} A_{0}^{\prime \prime}
$$

and

$$
g_{00}=\xi_{0}^{\prime} \xi_{0}^{\prime \prime}, \quad g_{11}=\xi_{1}^{\prime} \xi_{1}^{\prime \prime}, \quad g_{10}=\xi_{1}^{\prime} \xi_{0}^{\prime \prime} .
$$

The corresponding MCFs are plotted in Figure 16. For large and highly divergent supergranular cells (Figure 16(c)) the MCFs are similar to the MCFs for the nondiscretized parameters. However, both the maximum and the first minimum are shifted to larger distances and are more pronounced for areas than for maximum divergences and the shift between the two curves is slightly larger. The minimum of the curve for $g_{11}$ is even hardly detectable. This means that a regular ordering of strong divergence centers is clearly excluded. By applying $f_{00}$ and $g_{00}$ (Figure 16(b)) the heights of the first maxima as well as the depths of the first minima are more pronounced than for $f_{11}$ and $g_{11}$. This is due to the finite resolution of the analyzed data, which results in a higher complexity of the shapes of large cells than that of small ones. For $f_{00}$ even a shallow secondary maximum at $r=45 \mathrm{Mm}$ might be guessed. This result indicates a dense packing of small cells. Surprisingly, the shift between the two curves is opposite to that for larger cells. Finally, by applying $f_{10}$ and $g_{10}$ (Figure 16(d)), 
the obtained MCFs are again similar to those of the nondiscretized ones. Even the position of the maxima and minima are almost identical. Thus, the probability that a large and strong divergence center is surrounded by small and weakly divergent structures (or vice versa) is not different from the probability of finding equally large and divergent structures at the same distances.

\section{Conclusions}

The application of helioseismic methods to study the supergranulation phenomenon makes possible a statistical analysis of the structure of supergranulation. We have analyzed a sample of more than $10^{5}$ individual cells and several $10^{3}$ lifetime histories. The inferred mean cell diameter of $27.1 \mathrm{Mm}$ is in good agreement with former helioseismic studies (del Moro et al., 2004) and the local correlation tracking of granulation (November, 1994), but it is considerably larger than that found in works based on the analysis of SOHO/MDI Dopplergrams (DeRosa and Toomre, 2004) and Ca II K filtergrams (Hagenaar, Schrijver, and Title, 1997). Hagenaar, Schrijver, and Title (1997) explained the systematically smaller cell sizes obtained from Ca II K filtergrams by the fact that autocorrelation (AC) methods preferentially select larger cells. Much earlier, Singh and Bappu (1981) already obtained a mean cell size of $23 \mathrm{Mm}$ from Ca II K filtergrams and $32 \mathrm{Mm}$ with an AC method. In the present study a helioseismic cross-correlation method was applied to detect divergence centers. The obtained sizes are comparable with results from AC studies.

Schrijver, Hagenaar, and Title (1997) and Hagenaar, Schrijver, and Title (1997) have analyzed the distribution of supergranular cell sizes in the context of a Voronoi tessellation. They found a good agreement with the right-asymmetric shape of a Voronoi distribution. In the present study the obtained distribution of cell sizes is also right-asymmetric. However, it differs from that presented by Hagenaar, Schrijver, and Title (1997) since we find a minimum supergranular cell size of approximately $250 \mathrm{Mm}^{2}$ whereas they did not find a gap at small scales. This difference can be explained by the fact that we applied a noise filter with a cutoff at $\ell=250$. In contrast to these results, the size distribution found by del Moro et al. (2004) suggests a left-asymmetric shape.

From an analysis of the location of the cells on the solar disk using pair and mark correlation functions, we find some evidence that the supergranular cell locations represent a soft-core field, which has its closest analog of a field of nonoverlapping circles with variable diameters. The PCFs show only one conspicuous maximum and a weakly pronounced minimum. The results presented in Berrilli et al. (2004) are in disagreement with this result since in their analysis of PCFs they assume a hard-sphere random close packing model. The corresponding PCF shows several peaks, which can be roughly approximated by a damped cosine.

The inferred lifetime distributions are roughly exponential with mean decay times between 1.59 days (3D segmentation) and 1.83 days (feature tracking). These numbers are again larger than those obtained in most of the former studies, which are in a range around 20 hours (DeRosa and Toomre, 2004; del Moro et al., 2004). However, the analysis of Beck and Duvall (2001) gives a lifetime distribution comparable to the one derived here.

The temporal evolution of individual supergranular cells shows rather smooth histories from birth until death by dissolution. This is a little different from the findings of DeRosa and Toomre (2004), who stated that only $35 \%$ of the supergranular cells do not interact with adjacent structures during their existence. This difference may be due to the less-than-ideal temporal resolution of our data (12 hours versus 31 minutes for DeRosa and Toomre, 2004). 
The present study and the studies of Berrilli et al. (2004) and del Moro et al. (2004) have relied on similar data sets obtained from time - distance helioseismology. The two approaches, however, show significant differences in the distributions, lifetimes, and PCFs. This may be due to slightly different analyses or to differences in the computation of the divergence maps (here we applied a dispersion correction before measuring the travel times). The results presented in this paper are perfectly consistent with the findings of Gizon, Duvall, and Schou (2003), which also apply to the current data set.

Improvements are expected from the development of reliable inversions of the traveltime measurements (Jackiewicz et al., 2007; Jackiewicz, Gizon, and Birch, 2008). A better spatial sampling and, more importantly, a better temporal sampling will benefit studies of supergranulation evolution. We also plan to study a much longer data set to search for solarcycle variations in the structure of supergranulation, as hinted at in the preliminary analysis of Gizon and Duvall (2004).

Acknowledgements This paper is based on data from the Solar and Heliospheric Observatory, a project of international cooperation between ESA and NASA. The authors are grateful to an anonymous referee for careful reading and valuable comments.

Open Access This article is distributed under the terms of the Creative Commons Attribution Noncommercial License which permits any noncommercial use, distribution, and reproduction in any medium, provided the original author(s) and source are credited.

\section{References}

Alissandrakis, C.E., Dialetis, D., Tsiropoula, G.: 1987, Astron. Astrophys. 174, 275.

Beck, J.G., Duvall, T.L.: 2001, In: Wilson, A. (ed.) Helio- and Asteroseismology at the Dawn of the Millenium, Proc. SOHO 10/GONG 2000 Workshop SP-464, ESA, Noordwijk, 577.

Berrilli, F., Ermolli, I., Florio, A., Pietropaolo, E.: 1999, Astron. Astrophys. 344, 965.

Berrilli, F., del Moro, D., Conssolini, G., Pietropaolo, E., Duvall, T.L., Kosovichev, A.G.: 2004, Solar Phys. 221, 33 .

Braun, D.C., Birch, A.C., Lindsey, C.: 2004, In: Danesy, D. (ed.) Helio- and Asteroseismology: Towards a Golden Future, Proc. SOHO 14/GONG 2004 Workshop SP-559, ESA, Noordwijk, 337.

Crouch, A.D., Charbonneau, P., Thibault, K.: 2007, Astrophys. J. 662, 715.

del Moro, D., Berrilli, F., Duvall, T.L., Kosovichev, A.G.: 2004, Solar Phys. 221, 23.

DeRosa, M.L., Toomre, J.: 2004, Astrophys. J. 616, 1242.

Duvall, T.L., Gizon, L.: 2000, Solar Phys. 192, 177.

Gizon, L., Birch, A.C.: 2005, Living Rev. Solar Phys. 2(6). http://www.livingreviews.org//rsp-2005-6.

Gizon, L., Duvall, T.L.: 2004, In: Stepanov, A.V., Benevolenskaya, E.E., Kosovichev, A.G. (eds.) Multi Wavelength Investigations of Solar Activity, IAU Symp. 223, Cambridge Univ. Press, Cambridge, 41.

Gizon, L., Duvall, T.L., Schou, J.: 2003, Nature 421, 43.

Hagenaar, H.J., Schrijver, C.J., Title, A.M.: 1997, Astrophys. J. 481, 988.

Hart, A.B.: 1956, Mon. Not. Roy. Astron. Soc. 116, 38.

Hathaway, D.H., Beck, J.G., Bogart, R.S., Bachmann, K.T., Khatri, G., Petitto, J.M., Han, S., Raymond, J.: 2000, Solar Phys. 193, 299.

Hirzberger, J.: 2002, Astron. Astrophys. 392, 1105.

Hirzberger, J., Bonet, J.A., Vázquez, M., Hanslmeier, A.: 1999, Astrophys. J. 515, 441.

Jackiewicz, J., Gizon, L., Birch, A.C.: 2008, Solar Phys. DOI:10.1007/s11207-008-9158-7.

Jackiewicz, J., Gizon, L., Birch, A.C., Thompson, M.J.: 2007, Astron. Nachr. 328, 234.

Kosovichev, A.G., Duvall, T.L.: 1997, In: Pijpers, F.P., Christensen-Dalsgaard, J., Rosenthal, C.S. (eds.) Proc. SCORe'96: Solar Convection and Oscillations and their Relationship, ASSL 225, Kluwer, Dordrecht, 241.

Leighton, R.: 1964, Astrophys. J. 140, 1547.

Leighton, R., Noyes, R., Simon, G.W.: 1962, Astrophys. J. 135, 474.

Leitzinger, M., Brandt, P.N., Hanslmeier, A., Pötzi, W., Hirzberger, J.: 2005, Astron. Astrophys. 444, 245.

Mandelbrot, B.: 1977, Fractals, Freeman, San Francisco. 
Meunier, N., Roudier, T., Tkaczuk, R.: 2007, Astron. Astrophys. 466, 1123.

Meunier, N., Tkaczuk, R., Roudier, T.: 2007, Astron. Astrophys. 463, 745.

Meunier, N., Tkaczuk, R., Roudier, T., Rieutord, M.: 2007, Astron. Astrophys. 461, 1141.

November, L.J.: 1994, Solar Phys. 154, 1.

Ploner, S.R.O., Solanki, S.K., Gadun, A.S.: 2000, Astron. Astrophys. 356, 1050.

Rieutord, M., Roudier, T., Malherbe, J.M., Rincon, F.: 2000, Astron. Astrophys. 357, 1063.

Rieutord, M., Roudier, T., Roques, S., Ducottet, C.: 2007, Astron. Astrophys. 471, 687.

Rimmele, T., Schröter, E.H.: 1989, Astron. Astrophys. 221, 137.

Roudier, T., Muller, R.: 1987, Solar Phys. 107, 11.

Roudier, T., Lignières, F., Rieutord, M., Brandt, P.N., Malherbe, J.M.: 2003, Astron. Astrophys. 409, 299.

Sánchez Cuberes, M., Bonet, J.A., Vázquez, M., Wittmann, A.D.: 2000, Astrophys. J. 538, 940.

Scherrer, P.H., Bogart, R.S., Bush, R.I., Hoeksema, J.T., Kosovichev, A.G., Schou, J., Rosenberg, W., Springer, L., Tarbell, T.D., Title, A., Wolfson, C.J., Zayer, I., MDI Engineering Team: 1995, Solar Phys. 162, 129.

Schrijver, C.J., Hagenaar, H.J., Title, A.M.: 1997, Astrophys. J. 475, 328.

Shine, R.A., Simon, G.W., Hurlburt, N.E.: 2000, Solar Phys. 193, 313.

Simon, G.W., Leighton, R.B.: 1964, Astrophys. J. 140, 1120.

Singh, J., Bappu, M.K.V.: 1981, Solar Phys. 71, 161.

Srikanth, R., Singh, J., Raju, K.P.: 2000, Astrophys. J. 534, 1008.

Stoyan, D., Stoyan, H.: 1994, Fractals, Random Shapes and Point Fields, Wiley, Chichester.

Woodard, M.F.: 2002, Astrophys. J. 565, 634.

Zhao, J., Kosovichev, A.G.: 2003, In: Sawaya-Lacoste, H. (ed.) Local and Global Helioseismology: The Present and Future, Proc. SOHO 12/GONG 2002 Workshop SP-517, ESA, Noordwijk, 417. 